\title{
Face Detection and Recognition in Digital Images using Transform Domain Techniques
}

\author{
T. K. Sarode \\ $\mathrm{PhD}, \mathrm{T}$ hadomal Shahani College of Engg. \\ University of Mumbai, \\ Mumbai- 50,India.
}

\author{
Prachi Patil \\ Fr. Conceicao Rodrigues College of Engg. \\ University of Mumbai. \\ Mumbai- 50,India.
}

\begin{abstract}
Face Recognition is a computer application that is drawing much attention in the computer society in the areas like network security, privacy, video conferencing and content indexing and retrieval. These systems should be able to correctly detect and recognize different faces from the images. Many face recognition algorithms have been proposed that help faces to be identified on systems. The aim of the proposed system is to improvise by extracting the face region before feature extraction is done. Any image will be subjected to different lightening positions and intensities. Hence the system recognizes the face region using $\mathrm{YCbCr}$ color space. This paper also compares the results of different transform domain techniques applied further in the process.
\end{abstract}

\section{General Terms}

Security, Biometric system.

\section{Keywords}

Face Detection, skin detection, transform domain techniques.

\section{INTRODUCTION}

Given an image, the goal of any face detection and recognition system is to identify the correct location of the face in the image and match them with faces in the database.

In real scenarios, the images not only contain faces but also other objects. The effect of illumination and position of other background images makes face detection difficult.

Face Recognition and Detection system proposed here consists of three phases.

1. Face Detection.

2. Feature Extraction

3. Face Recognition.

In the proposed system, the face region will be extracted from the input image using $\mathrm{YCbCr}$ color model. In second phase, feature vector will then be calculated using transform domain techniques like DCT, Walsh, Haar, Kekre's transforms. For face recognition phase, the feature vector will then be compared with the feature vectors for images stored in database. The image showing the minimum error will be the output of recognition process

This paper shows a comparison between recognition rates using different transform domain techniques and vector quantization techniques.

Figure 1 shows the steps involved in the proposed system. 


\section{FACE DETECTION}

The first step in face detection is to identify the face regions in the entire image[1]. To make the proposed system independent of luminescence effect, faces are identified using YCbCr color space. The $\mathrm{Cb}$ and $\mathrm{Cr}$ components help to identify skin regions in input image based on thresholds. The result of this is a binary image which identifies the position of different skin pixels.

The pixels are marked as skin pixels which fall in the range of set thresholds. The threshold values for the range of $\mathrm{CB}$ and $\mathrm{Cr}$ are identified using experimental results.

$\mathrm{r} 1<\mathrm{Cr}(\mathrm{i}, \mathrm{j})<\mathrm{r} 2$

$\mathrm{r} 3<(\mathrm{Cb}(\mathrm{i}, \mathrm{j})+0.6 * \mathrm{Cr}(\mathrm{i}, \mathrm{j}))<\mathrm{r} 4$

$\mathrm{r} 5<\mathrm{Cb}(\mathrm{i}, \mathrm{j})<\mathrm{r} 6$

where $\mathrm{i}, \mathrm{j}$ are current pixel position and $1<\mathrm{i}, \mathrm{j}<\mathrm{N}, \mathrm{N}$ is number of pixels in rows and columns. $\mathrm{r} 1$ to $\mathrm{r} 5$ are positive integer values which vary for different skin tones.
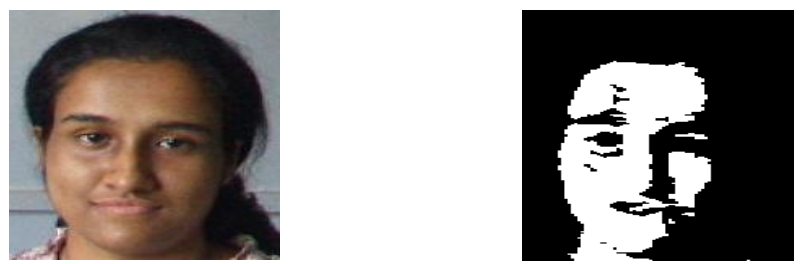

Fig 2. shows the sample input image and result of skin region detection.

The steps for face region detection are as follows.

1. Build the skin color model ( $\mathrm{YCbCr}$ space).

2. Perform skin segmentation using appropriate thresholds on $\mathrm{Cb}$ and $\mathrm{Cr}$ components using above equation.

3. Extract the face region using region identified in step 2.
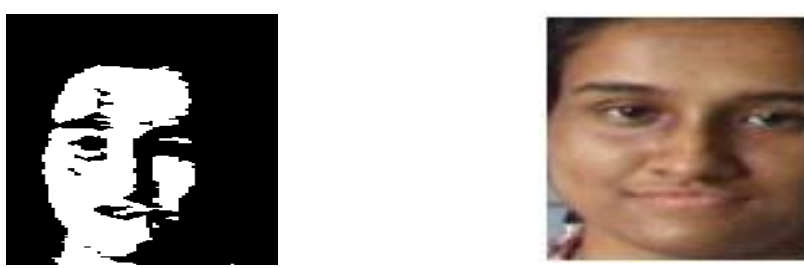

Fig 3. shows the face region extracted from binary image.

The result of this stage is given as an input to face recognition process.

\section{FACE RECOGNITION}

Once the face region has been identified, the next step is to correctly identify the face. To match among different faces detected, feature vectors are calculated for each image using 2D transform domain techniques and 1D transform domain techniques.

The feature vector of input image is them compared with feature vectors of other images in database. The image with minimum error value is considered to be the correct match.

$$
\mathrm{MSE}=\frac{\sum_{\mathrm{M}, \mathrm{N}}\left[\mathrm{I}_{1}(\mathrm{~m}, \mathrm{n})-\mathrm{I}_{2}(\mathrm{~m}, \mathrm{n})\right]^{2}}{\mathrm{M} * \mathrm{~N}}
$$

Where $\mathbf{M}$ and $\mathbf{N}$ are the number of rows and columns respectively in the input images.

The steps for feature extraction are as follows:

1. Calculate the feature vector using various transforms on extracted face. Store the features of input image and various other images in database.

2. Calculate the mean square error to find the difference between feature vectors of extracted face from input image and faces in database. If both the features vectors matches, then face is correctly identified.

The following subsection discusses the different techniques applied to calculate feature vector.

\subsection{Feature Vector using Transform domain techniques.}

2D Transform domain techniques like DCT[2], Walsh[3], Haar and Kekre's[4] are applied over the output of face detection phase.

Feature vectors are even calculated by using row mean and column mean[5][6][7][8]. The row mean vector is the set of averages of the intensity values of the respective rows. The column mean vector is the set of averages of the intensity values of the respective columns

Row Mean Vector $=$

[Avg(Row1), Avg(Row2),..... Avg(Row n)]

Column Mean Vector $=$

$[\operatorname{Avg}(\operatorname{Col~1)}), \operatorname{Avg}(\operatorname{Col} 2), \ldots \ldots \ldots \operatorname{Avg}(\operatorname{Col} n)]$

A new feature vector is calculated by arranging the row mean vector in first column and column mean vector in second column in the resultant vector.

Row-Column Mean Vector $=$

[Row_mean_Vector Column_Mean_Vector]

Feature vector of varying sizes are calculated by considering the partial co-efficients from top left corner of 2D coefficients matrix and different algorithms are compared based on these feature vectors.

The error value is calculated using mean squared error metric. 


\section{RESULTS}

Table 1 shows the results of 2D transforms applied over Indian Face Database of 1000 test images.

Table 1. Shows accuracy (\%) for different feature vector sizes when 2D transforms are applied to extracted faces.

\begin{tabular}{|c|c|c|c|c|}
\hline & \multicolumn{4}{|c|}{ Transform Domain Techniques 2D } \\
\cline { 2 - 5 } & DCT & WALSH & HAAR & KEKRE \\
\hline $\mathbf{4} * \mathbf{4}$ & 83.16 & 15.3 & 15.3 & 15.3 \\
\hline $\mathbf{8} * \mathbf{8}$ & 79.08 & 21.93 & 21.93 & 21.93 \\
\hline $\mathbf{1 6} * \mathbf{1 6}$ & 78.57 & 32.14 & 32.14 & 22.95 \\
\hline $\mathbf{3 2} * \mathbf{3 2}$ & 78.06 & 42.34 & 42.34 & 32.14 \\
\hline $\mathbf{6 4} * \mathbf{6 4}$ & 78.06 & 55.61 & 55.61 & 42.85 \\
\hline $\mathbf{1 2 8} * \mathbf{1 2 8}$ & 78.06 & 68.87 & 68.87 & 47.74 \\
\hline $\mathbf{2 5 6} * \mathbf{2 5 6}$ & 77.55 & 77.55 & 77.55 & 37.75 \\
\hline
\end{tabular}

It can be seen that the recognition rate increases as the feature vector sizes increases whereas the recognition rate in DCT increases when feature vector size is minimum. The highest recognition rate of $83.16 \%$ for feature vector size $4 * 4$ is achieved using DCT transform amongst all other techniques. $77.55 \%$ accuracy is achieved using Haar and Walsh transform for feature vector size $256 * 256$ and $47.74 \%$ accuracy is achieved for Kekre transform using vector size 128*128.

Results of applying 1D transforms (Row Mean) technique are tabulated below in table 2 .

Table 2. Shows accuracy (\%) for different feature vector sizes for 1D transforms applied on row mean of extracted faces.

\begin{tabular}{|c|c|c|c|c|}
\hline \multirow{2}{*}{} & \multicolumn{5}{|c|}{$\begin{array}{c}\text { Transform Domain Techniques 1D } \\
\text { (Row Mean) }\end{array}$} \\
\cline { 2 - 5 } & DCT & WALSH & HAAR & KEKRE \\
\hline $\mathbf{4} * \mathbf{1}$ & 43.87 & 6.63 & 37.75 & 18.36 \\
\hline $\mathbf{8} * \mathbf{1}$ & 56.63 & 8.16 & 53.06 & 19.89 \\
\hline $\mathbf{1 6} * \mathbf{1}$ & 58.16 & 14.28 & 57.14 & 22.95 \\
\hline $\mathbf{3 2} * \mathbf{1}$ & 56.63 & 16.83 & 57.14 & 30.1 \\
\hline $\mathbf{6 4} * \mathbf{1}$ & 56.63 & 27.04 & 56.63 & 42.34 \\
\hline $\mathbf{1 2 8} * \mathbf{1}$ & 56.63 & 37.24 & 56.63 & 57.14 \\
\hline $\mathbf{2 5 6} * \mathbf{1}$ & 56.63 & 56.63 & 56.63 & 56.63 \\
\hline
\end{tabular}

It is observed that the recognition rate for DCT is $58.16 \%$ using feature vector size of $16^{*} 1$ is highest among all techniques. The recognition rates for other techniques increases with increase in feature vector sizes. For Walsh and Haar technique the highest recognition of $56.63 \%$ each is achieved for feature vector size of $256^{*} 1$ and Kekre transform shows a result of $57.14 \%$ at feature vector size $128 * 1$.

Table 3 represents results of applying 1D column mean technique over the images.

recognition rate is maximum when feature vectors are calculated using 2D-DCT transform. The performance is
Table 3. Shows accuracy ( $\%$ ) for different feature vector sizes for 1D transforms applied on column mean of extracted faces.

\begin{tabular}{|c|c|c|c|c|}
\hline \multirow{2}{*}{} & \multicolumn{4}{|c|}{$\begin{array}{c}\text { Transform Domain Techniques 1D } \\
\text { (Column Mean) }\end{array}$} \\
\cline { 2 - 5 } & DCT & WALSH & HAAR & KEKRE \\
\hline $\mathbf{1} * \mathbf{4}$ & 45.6 & 6.63 & 44.89 & 21.93 \\
\hline $\mathbf{1} * \mathbf{8}$ & 56.12 & 10.2 & 55.61 & 24.48 \\
\hline $\mathbf{1} \mathbf{1 6}$ & 58.16 & 11.22 & 57.14 & 27.04 \\
\hline $\mathbf{1} * \mathbf{3 2}$ & 59.18 & 18.87 & 58.16 & 34.18 \\
\hline $\mathbf{1} * \mathbf{6 4}$ & 58.67 & 28.57 & 58.67 & 45.91 \\
\hline $\mathbf{1} * \mathbf{1 2 8}$ & 58.16 & 41.32 & 58.67 & 52.55 \\
\hline $\mathbf{1} \mathbf{2 5 6}$ & 58.67 & 58.67 & 58.67 & 58.67 \\
\hline
\end{tabular}

It is observed that DCT shows the highest accuracy of 59.18\% for vector size $1 * 32$ followed by Walsh, Haar and Kekre transforms with accuracy of $58.67 \%$ for vector size $1 * 256$.

Results of applying 1D transform (Row-Column Mean technique) are shown below in table 4 .

Table 4. Shows accuracy $(\%)$ for different feature vector sizes for 1D transforms applied on row-column mean of extracted faces.

\begin{tabular}{|c|c|c|c|c|}
\hline \multirow{2}{*}{} & \multicolumn{5}{|c|}{$\begin{array}{c}\text { Transform Domain Techniques 1D } \\
\text { (Row-Column Mean) }\end{array}$} \\
\cline { 2 - 5 } & DCT & WALSH & HAAR & KEKRE \\
\hline $\mathbf{4} * \mathbf{2}$ & 64.79 & 9.69 & 60.71 & 28.57 \\
\hline $\mathbf{8} * \mathbf{2}$ & 74.48 & $11 . .73$ & 73.97 & 32.14 \\
\hline $\mathbf{1 6} * \mathbf{2}$ & 75.51 & 17.34 & 75.51 & 43.36 \\
\hline $\mathbf{3 2} * \mathbf{2}$ & 75.51 & 25 & 76.02 & 48.46 \\
\hline $\mathbf{6 4} * \mathbf{2}$ & 75 & 39.79 & 75.51 & 55.61 \\
\hline $\mathbf{1 2 8} * \mathbf{2}$ & 75 & 49.48 & 75 & 68.87 \\
\hline $\mathbf{2 5 6} * \mathbf{2}$ & 75 & 75 & 75 & 75 \\
\hline
\end{tabular}

From the results it is observed that Haar transform gives maximum accuracy of $76.02 \%$ for feature vector size $32 * 2$. DCT has a maximum recognition rate of $75.51 \%$ for vector size $16 * 2$ followed by Walsh and Haar transform with accuracy of $75 \%$ for vector size $256 * 2$.

\section{CONCLUSION}

In this paper 1D and 2D transform domain techniques are applied for feature extraction in the images. Transforms like DCT, Walsh, Haar and Kekre's are considered and compared with all coefficients and partial coefficients. The system calculates feature vectors of varying sizes by considering coefficients from top left corner of coefficients matrix. 1D feature vectors are calculated using row mean, column mean and combination of row and column mean techniques.

The proposed system compares 1000 images in Indian face database. From the observations it can be stated that after applying different transform domain techniques, the maximum for minimum feature vector size of $4 * 4$ in case of DCT transform. Whereas the Walsh, Haar and Kekre 
transform results are maximum when feature vector size is maximum.

The application of row mean and column mean techniques does not out perform in comparison to 2D transforms. Whereas the recognition rates after combining row mean and column mean are good in comparison to applying row mean and column mean individually.

The highest recognition rate in both the result set is $83.16 \%$ using 2D DCT transform with the smallest feature vector size of $4 * 4$, giving up the advantage.

\section{REFERENCES}

[1] Mohamed Berbar, Hamdy Kelash, "Faces and Facial Features Detection in Color Images", GMAI'06, pp. 1-6, 2006.

[2] Andrew B. Watson, "Image compression using the Discrete Cosine Transform", Mathematica journal, 4(1), pp. 81-88, 1994.

[3] Dr. H. B. Kekre, Sudeep Thepade, Archana Athawale, Anant Shah, Prathamesh Verlekar, Suraj Shirke. "Walsh Transform over Row Mean and Column Mean using Image Fragmentation and Energy Compaction for Image Retrieval". / (IJCSE) International Journal on Computer Science and Engineering, Vol. 02, No.01S, 2010, 47-54.

[4] "Image Retrieval using Non-Involutional Orthogonal Kekre's Transform", International Journal of
Multidisciplinary Research and Advances in Engineering (IJMRAE), Ascent Publication House, 2009, Volume 1, No.I, pp 189-203, 2009.

[5] H.B.Kekre, Tanuja Sarode, Sudeep D. Thepade, "DCT Applied to Row Mean and Column Vectors in Fingerprint Identification", In Proceedings of International Conference on Computer Networks and Security (ICCNS), 27-28 Sept. 2008, VIT, Pune.

[6] H.B.Kekre, S. D. Thepade, A. Athawale, A. Shah, P. Verlekar, S. Shirke. "Performance Evaluation of Image Retrieval using Energy Compaction and Image Tiling over DCT Row Mean and DCT Column Mean". Springer-International Conference on Contours of Computing Technology (Thinkquest-2010), Babasaheb Gawde Institute of Technology, Mumbai, 2010.

[7] H.B.Kekre, Sudeep D. Thepade, "Improving the Performance of Image Retrieval using Partial Coefficients of Transformed Image", International Journal of Information Retrieval (IJIR), Serials Publications, Volume 2, Issue 1, 2009, pp. 72-79.

[8] H. B. Kekre, S. Thepade, A. Maloo. "Performance Comparison of Image Retrieval Using Fractional Coefficients of Transformed Image Using DCT, Walsh, Haar and Kekre's Transform”. CSC-International Journal of Image processing (IJIP), 4(2)142-155, 2010. 